\title{
Conférence inaugurale
}

Les enjeux des maladies

rares dans les évolutions

du système de santé

français

\section{Dominique Péton-Klein}

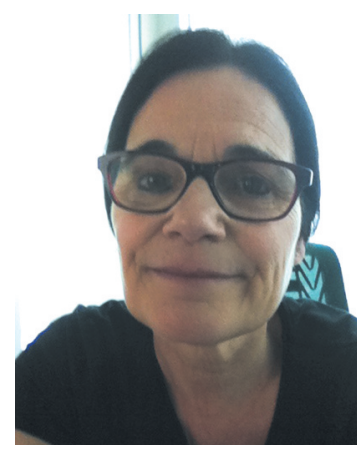

Secrétaire générale

PNMR 2011-2014 précédemment assurées par le Dr Alain Garcia. Parmi ces fonctions figurent le suivi, la coordination et l'évaluation du second plan national maladies rares.

Pour ce troisième congrès, vous avez souhaité aborder la question des enjeux des maladies rares dans les évolutions du système de santé français. Cette question est particulièrement légitime dans le contexte actuel de réflexion sur les évolutions souhaitées de la politique de santé. Au préalable, je rappellerai quelques fondamentaux sur ce qui a déjà été fait, ce qui est en cours, ce qui permettra de situer où nous en sommes au regard des enjeux posés dans le cadre de la stratégie nationale de santé (SNS), qui sont actuellement en cours de réflexion.

Vous connaissez tous les grandes étapes de ce dossier. Les associations ont en effet largement contribué à son émergence. Il est néanmoins nécessaire de rappeler que le $1^{\text {er }}$ plan national maladies rares (PNMR) 2005-2008 figurait parmi les cinq priorités de santé publique, notamment dans la loi du 9 août 2004 relative à la politique de santé publique. L'un des objectifs de ce plan était de développer et renforcer les initiatives déjà prises, et d'en accroître la synergie.

À mon arrivée, j'ai pris connaissance de plusieurs rapports, et notamment du rapport d'évaluation du Haut Conseil de la santé publique (HCSP) d'avril 2009. Véritable mine d'informations, ce document m'a permis très rapidement de rentrer dans le vif du sujet. Quelques propos de ce rapport décrivent très bien la situation, et apportent un éclairage sur l'architecture

mise en place - et au-delà sa reproductibilité. Les auteurs de ce rapport expliquent notamment que «de l'analyse du plan naît une certaine satisfaction, conforme au jugement globalement positif exprimé par ses principaux acteurs. Cette impression générale ne doit toutefois masquer ni une réalité plus contrastée ni les difficultés à venir: nous ne sommes qu'au début de la construction d'un nouveau segment de la politique de santé. Le premier Plan nous lègue une infrastructure de l'édifice, mais si l'effort n'est pas soutenu, ce socle deviendra rapidement inutile». Cet effort a bien été soutenu dans le second plan, mais la maxime énoncée dans le rapport reste d'actualité.

À l'issue de ce premier plan ont été soulignées l'organisation et la structuration mises en place pour la prise en charge de ces maladies, et notamment l'organisation avec les centres de référence maladies rares, les centres de compétences, le lien recherche, clinique et soins. Les auteurs observent également que les maladies rares sont en voie de constituer un modèle conceptuel et méthodologique pour l'ensemble des sciences de la santé. Ils donnent plusieurs raisons.

- Parce qu'elles sont l'exemple même du continuum dans la rigueur entre la recherche fondamentale et la recherche clinique. À ce titre, elles ont largement bénéficié des progrès de la biologie moléculaire.

- Parce que les maladies rares ont bouleversé les pratiques médicales et le rapport médecin-malade. II faut souligner le rôle éminent joué par les associations, car leur rareté est source d'enseignement. Le caractère européen des fédérations et des associations, et le nombre de groupes de travail de médecins et de chercheurs travaillant sur les mêmes maladies l'attestent. 
- Parce que les efforts qui les concernent s'inscrivent dans la durée. La plupart des maladies rares entraînent en effet un long dialogue entre un malade et un système de santé dans son ensemble. À ce titre, elles agissent comme un révélateur des défauts et des progrès de toutes les prises en charge: diagnostic, insertion scolaire, transition enfant-adulte, aides sociales, conseil génétique, milieu professionnel, solidarité citoyenne. La France a été pionnière dans la structuration de ce domaine en étant le premier pays de I'Union européenne à se doter d'un plan national en 2004 et d'un second plan à partir de 2011 . La plupart des autres pays sont encore en phase de réflexion ou de rédaction d'un premier plan, alors que la recommandation de juin 2009 du conseil de l'Union européenne était que chaque pays adoptât avant la fin 2013 un plan ou une stratégie pour les maladies rares.

Ce premier plan a permis d'édifier une infrastructure essentielle et jugée positive; un des enjeux majeurs du second plan est de consolider ces acquis et d'assurer une prise en charge aussi adaptée que possible. Ce second plan a été construit en tenant compte de ces recommandations. Quelques données à notre disposition doivent être rappelées. En 2009, environ $6 \%$ de la population mondiale - soit 4 millions de personnes en France et 24 millions en Europe - était atteinte d'une maladie rare. L'enquête qualitative menée par le HCSP en 2009 auprès de patients atteints de maladies rares avait relevé trois points majeurs:

- les difficultés considérables rencontrées par les patients dans leur vie quotidienne;

- l'importance capitale pour le patient et son entourage de la reconnaissance sociale de la maladie;

- le grand intérêt des centres de référence pour le diagnostic, le suivi et l'information, mais l'existence de grandes inégalités dans la prise en charge, avec un déficit dans tous les domaines quand il n'y a pas de centre de référence ou qu'il est loin.

Du rapport d'activités Maladies Rares info Services, je retiendrai deux éléments: l'errance diagnostique est de moins de trois ans - contre dix ans il y a encore quelques années, comme cela a été rappelé lors d'un congrès organisé par l'Agence régionale de santé (ARS) à Rennes la semaine dernière par les associations; l'importance du nombre de médecins consultés en l'absence de diagnostic à la naissance (50\% des participants à l'enquête ont déclaré avoir consulté deux à cinq médecins en cabinets libéraux, et autant en milieu hospitalier).

Quel bilan peut-on établir au terme des trois premières années du second plan? Je ne citerai que quelques exemples, puisqu'un bilan plus précis sur l'état d'avancement des différentes mesures et actions de ce plan est en cours de réflexion; il sera présenté à un comité de suivi et de prospective au début du mois de février 2014. Les exemples d'actions suivants apparaissent répondre au mieux à des interrogations découlant de l'évaluation du premier plan.

\section{La création des filières de santé maladies rares}

Cette mesure est fondamentale. Le premier plan nous a légué une infrastructure de centres de référence et de centres de compétences qui est enviée de beaucoup. II est essentiel de la consolider, de la rendre plus lisible pour les patients et les professionnels de santé, de l'évaluer et de la faire évoluer. Un certain nombre de dossiers ont été déposés suite à l'appel à projets filières lancé fin juillet 2013 ; ces dossiers sont en cours d'examen. Les projets seront examinés d'ici quelques semaines, pour une mise en œuvre effective prévue pour le début 2014. Les projets déposés traduisent un important travail de réflexion et de structuration des centres de référence, un important travail de pédagogie et de rapprochement de ces centres et des coordonnateurs de ces centres.

\section{La révision du processus de labellisation} des centres de référence et le suivi de leur activité Le nouveau référentiel d'évaluation des centres de référence sera publié sur le site de la Haute Autorité de santé (HAS). Par ailleurs, nous travaillons sur un rapport d'activité que devront renseigner annuellement tous les centres de référence. $\varepsilon$ n cours de finalisation, ce document devrait être disponible au premier semestre 2014. Ces deux chantiers complémentaires seront suivis par le groupe permanent, qui sera installé le 18 décembre 2013 - dans un premier temps pour étudier les dossiers des filières, et dans un second temps pour étudier le renouvellement du processus de labellisation des centres de référence.

\section{La mise en place d'une Banque nationale de données maladies rares (BNDMR)}

Dans le premier axe du plan, le HCSP définissait plusieurs problématiques, dont la création d'une base de données. Le développement d'une BNDMR prévu dans le second plan doit répondre à ces recommandations. Ses objectifs sont de mettre en place un système d'information unique et partagé entre les centres de référence et les centres de compétences, de faire avancer les connaissances sur l'histoire naturelle des maladies, de documenter les modes de prise en charge, de décrire les demandes de soins, de produire des connaissances médico-économiques, et de coupler un profil phénotypique avec des données issues de la génomique. La mise en place de cet outil nous servira incontestablement à avoir une bien meilleure visibilité du parcours des patients, permettant ainsi d'améliorer le dispositif de soins et de prise en charge. L'objectif est de mettre en place la BNDMR dans le courant 2014.

La semaine dernière, nous avons tenu le $4^{\mathrm{e}}$ comité de suivi et de prospective, qui a porté sur les thèmes des médicaments, de la prise en charge sanitaire et de l'accompagnement médico-social. Certaines initiatives nous ont été exposées, comme celle de l'Assistance Publique-Hôpitaux de Paris visant à favoriser des 
échanges entre les centres de référence, les centres de compétences et les maisons départementales des personnes handicapées, ainsi qu'un travail de réflexion sur le certificat médical.

Ces quelques exemples montrent que le second plan s'est bien inscrit dans la poursuite des actions passées. Qu'en sera-t-il dans la perspective de la nouvelle stratégie nationale de santé (SNS) ? Celle-ci a été annoncée par le Premier ministre le 8 février 2013 avec pour ambitions de définir un cadre de l'action publique pour les années à venir, combattre les injustices, les inégalités de santé et d'accès au système de soins, et mieux adapter le système de santé français aux besoins des patients. Marisol Touraine, ministre des Affaires sociales et de la Santé, et Geneviève Fioraso, ministre de l'Enseignement supérieur et de la Recherche, ont présenté le 23 septembre 2013 les grands objectifs de la SNS. Ses trois axes principaux sont les suivants:

- prioriser la prévention sur le curatif et agir sur les déterminants de santé ;

- mieux organiser les soins pour les patients, et garantir l'égalité d'accès en privilégiant une logique territoriale ;

- miser sur la déconcentration et renforcer la démocratie sanitaire.

La SNS définit les orientations d'une politique globale; la prise en charge des maladies rares en fait partie, et les priorités définies ne pourront que permettre d'améliorer un certain nombre d'insuffisances encore observées. Pour d'autres actions en cours ou achevées, ces priorités s'inscrivent d'ores et déjà dans cette politique.

Pour illustrer l'implication du champ des maladies rares dans la SNS, j'ai relevé quelques priorités. Cette stratégie établit comme priorité une politique forte sur le premier recours. II importe que les professionnels de premier recours soient formés et informés, et acquièrent le réflexe de penser systématiquement à une possible maladie rare. Orphanet, créé en 1997, est aujourd'hui la source d'informations incontournable pour les professionnels de santé et les patients. On peut se poser la question du recours et des systèmes d'information. Ces derniers existent, mais il importe de mieux les faire connaître auprès des médecins de premier recours. Cet enjeu important devra faire l'objet de réflexion à l'occasion notamment des forums et des débats qui auront lieu dans les différentes régions.

La SNS souligne la priorité de mettre en place un appui aux équipes de proximité. Des exemples dans d'autres domaines comme les maisons pour l'autonomie et l'intégration des malades d'Alzheimer (MAIA) et les réseaux santé. L'idée est de s'approprier ce type d'organisation, qui peut être spécifique à un handicap ou une pathologie, et de voir si son intégration est possible dans d'autres champs comme celui des maladies rares. On ne peut en effet penser et créer des organisations propres à chaque maladie ou groupe de maladies; il importe de capitaliser sur l'existant, et de l'adapter en élargissant ses missions. La Caisse nationale de sécurité sociale (CNSS) rappelle également l'intrication forte de la recherche et de l'innovation. Lors de son discours du 23 septembre 2013, la ministre de l'Enseignement supérieur et de la Recherche a déclaré que la SNS devait nous permettre de faire face dans les années à venir aux profondes transformations du champ de la biologie et de la médecine, soulignant que l'enjeu est de garder ce niveau d'excellence et d'assurer le continuum allant de la recherche la plus fondamentale à la recherche translationnelle - témoignant du passage de la recherche en amont jusqu'aux soins. L'axe recherche du PNMR en cours traduit explicitement cette volonté. L'évaluation devra nous permettre d'en tirer les premiers enseignements, et d'adapter éventuellement les pratiques.

Enfin, la SNS détermine comme priorité de s'appuyer sur une gouvernance associant tous les acteurs, sur un service public territorial rénové. Notre ministre a déclaré : «Avec l'ensemble des acteurs de santé et de leur territoire, et en soutenant leurs initiatives, les agences régionales de santé doivent favoriser la réorganisation des soins de premier recours, développer la prévention et les prises en charge médico-sociales, etc. » L'implication des ARS dans le champ des maladies rares est nécessaire et importante, ces actions s'inscrivant dans une démarche de prévention, de soins et de prise en charge médico-sociale.

Lors du dernier comité de suivi et de prospective, nous avons évoqué la mise en place souhaitée de services territoriaux destinés à faire ce lien entre des acteurs, et rendant plus fluide l'ensemble du parcours des personnes malades. Quelques expériences et projets sont en cours de réflexion dans ce domaine.

Les chantiers à venir sont nombreux, et vont s'ouvrir dans le cadre de ces nouvelles orientations. Les maladies rares feront partie intégrante de cet avenir. De novembre à février se tiendront sur tout le territoire des forums régionaux de santé. Le premier s'est tenu à Rennes le 19 novembre. De nombreux forums sont d'ores et déjà annoncés, notamment en région ProvenceAlpes-Côte-d'Azur. II importe que vous y soyez présents. Récemment nommée, j'ai été amenée à rencontrer un certain nombre de professionnels, de représentants, d'institutions et d'associations. Ces rencontres m'ont permis d'une part de mesurer le chemin parcouru, et d'autre part d'apprécier l'implication majeure de chacun d'entre vous. Je salue votre engagement et votre travail, et vous assure que nous sommes et serons à vos côtés au quotidien. $\diamond$

\section{LIENS D'INTÉRÊT}

L'auteur déclare n'avoir aucun lien d'intérêt concernant les données publiées dans cet article. 